\title{
Stress-induced cortical dopamine response is altered in subjects at clinical high risk for psychosis using cannabis
}

\author{
Christin Schifani $^{1}$ Jens Pruessner ${ }^{2,3}$ Huai-Hsuan Tseng ${ }^{1}$ Naren Rao ${ }^{1}$ \\ Abanti Tagore $^{1,4}$ Alan A. Wilson ${ }^{1}$ Sylvain Houle ${ }^{1}$ Pablo M. Rusjan ${ }^{1}$ \\ Romina Mizrahi ${ }^{1,5,6}$
}

${ }^{1}$ Research Imaging Centre, Centre for Addiction and Mental Health, Toronto, Ontario, Canada

${ }^{2}$ Departments of Psychology, Psychiatry, Neurology and Neurosurgery, Douglas Institute, McGill University, Montreal, Quebec, Canada

${ }^{3}$ Department of Psychology, University of Constance, Constance, Germany

${ }^{4}$ Institute of Medical Science, University of Toronto, Toronto, Ontario, Canada

${ }^{5}$ Campbell Family Mental Health Research Institute, Centre for Addiction and Mental Health, Toronto, Ontario, Canada

${ }^{6}$ Department of Psychiatry, University of Toronto, Toronto, Ontario, Canada

\section{Correspondence}

Romina Mizrahi and Pablo M. Rusjan, PET Centre, Research Imaging Centre, Centre for Addiction and Mental Health, 250 College Street, Toronto, Ontario, Canada M5T 1R8. Email: romina.mizrahi@camhpet.ca; pablo. rusjan@camhpet.ca

Funding information

Canadian Institutes of Health Research

\begin{abstract}
Stress and cannabis use are risk factors for the development of psychosis. We have previously shown that subjects at clinical high risk for psychosis (CHR) exhibit a higher striatal dopamine response to stress compared with healthy volunteers (HV), with chronic cannabis use blunting this response. However, it is unknown if this abnormal dopamine response extends to the prefrontal cortex (PFC). Here, we investigated dorsolateral PFC (dIPFC) and medial PFC (mPFC) dopamine release using $\left[{ }^{11} \mathrm{C}\right]$ FLB457 positron emission tomography (PET) and a validated stress task. Thirty-three participants completed two PET scans (14 CHR without cannabis use, eight CHR regular cannabis users [CHR-CUs] and $11 \mathrm{HV}$ ) while performing a Sensory Motor Control Task (control scan) and the Montreal Imaging Stress Task (stress scan). Stress-induced dopamine release $\left(\triangle B P_{N D}\right)$ was defined as percent change in $D_{2 / 3}$ receptor binding potential between both scans using a novel correction for injected mass of $\left[{ }^{11} \mathrm{C}\right] \mathrm{FLB} 457 . \triangle B P_{\mathrm{ND}}$ was significantly different between groups in $\operatorname{mPFC}(F(2,30)=5.40$, .010), with CHR-CUs exhibiting lower $\triangle B P_{N D}$ compared with $C H R$ (.008). Similarly, salivary cortisol response $\left(\triangle A \cup C_{1}\right)$ was significantly lower in $\mathrm{CHR}-\mathrm{CU}$ compared with $\mathrm{CHR}(F(2,29)=5.08$, .013 ; post hoc .018) and positively associated with $\triangle B P_{N D}$. Furthermore, CHR-CUs had higher attenuated psychotic symptoms than $\mathrm{CHR}$ following the stress task, which were negatively associated with $\triangle B P_{N D}$. Length of cannabis use was negatively associated with $\triangle B P_{N D}$ in $\mathrm{mPFC}$ when controlling for current cannabis use. Given the global trend to legalize cannabis, this study is important as it highlights the effects of regular cannabis use on cortical dopamine function in high-risk youth.
\end{abstract}

KEYWORDS

cannabis, clinical high risk, dopamine, positron emission tomography, prefrontal cortex, stress

\section{INTRODUCTION}

Cannabis is one of the most widely used recreational drugs worldwide $^{1}$ and the most commonly used illicit drug in patients on the psychosis spectrum including those with schizophrenia ${ }^{2}$ and those at elevated risk. ${ }^{3}$ Longitudinal studies link cannabis use to a significantly increased risk of subsequent development of psychotic symptoms or psychotic illness (recently reviewed by Murray et $\mathrm{al}^{4}$ ). A recent 
meta-analysis found a dose-response relationship between higher cannabis use and increased risk for schizophrenia. The reported median odds ratio for the risk of schizophrenia and other psychosis-related outcomes for any cannabis use was 1.97 , whereas the odds ratio was 3.90 among the heaviest users. ${ }^{5}$ Further, psychotic patients using cannabis have earlier illness onset than non-users. ${ }^{6}$ However, little is known about the effects of cannabis on brain neurochemistry, and specifically about its impact on dopamine neurotransmission, which is important as schizophrenia presents with abnormal dopamine synthesis and release in striatal regions (recently reviewed by Weinstein et $\mathrm{al}^{7}$ ).

Drugs of abuse such as cocaine, amphetamine, methamphetamine, and alcohol are associated with decreased striatal dopamine release when used regularly. ${ }^{8,9}$ This has also been reported for individuals with cannabis dependence showing blunted dopamine release in striatum upon amphetamine challenge compared with non-users ${ }^{10}$ and for cannabis abusers showing a blunted dopamine response in striatum after methylphenidate challenge compared with non-users ${ }^{11}$ (but see also Mizrahi et $\mathrm{al}^{12}$; Urban et $\mathrm{al}^{13}$ ). Moreover, cannabis users at clinical high risk for psychosis (CHR) had lower stress-induced striatal dopamine release as compared with non-users, which was associated at trend level with the age of onset of cannabis use in associative striatum. ${ }^{14}$

The prefrontal cortex (PFC) is well known for its crucial role in planning, controlling, and directing behaviour in response to changing environmental demands. While the medial PFC (mPFC) is extensively connected to subcortical regions that generate emotional responses such as amygdala and hypothalamus, the dorsolateral PFC (dIPFC) is connected with sensory and motor cortices and is a key region to regulate cognitive demand. ${ }^{15}$ Cannabis use is associated with deficits in both emotional processing ${ }^{16}$ and neurocognitive function (recently reviewed by Volkow et $\mathrm{al}^{17}$ ), consistent with observations in patients with schizophrenia. ${ }^{18}$ Cognitive deficits in patients with first episode psychosis were further linked to a reduction in prefrontocortical dopamine release in response to an amphetamine challenge. ${ }^{19}$ Although our recent study reported no difference in prefrontocortical dopamine release in response to a stress challenge between patients with schizophrenia, CHR participants, and healthy volunteers (HV), we found that patients with schizophrenia had a disrupted PFC dopamine-stress response. ${ }^{20}$ Furthermore, we observed that $\mathrm{CHR}$ participants with higher distress and anxiety exhibited lower mPFC and dIPFC dopamine release. ${ }^{20}$

To date, no study examined the effect of regular cannabis use on cortical dopamine transmission in subjects at risk for developing psychosis such as $\mathrm{CHR}$. Therefore, the present study aimed to examine mPFC and dIPFC dopamine release in response to a psychosocial stress task in $\mathrm{CHR}$ with and without regular cannabis use and $\mathrm{HV}$ using a validated two-scan paradigm with $\left[{ }^{11} \mathrm{C}\right] \mathrm{FLB} 457$ positron emission tomography (PET). We hypothesized a lower PFC dopamine release in CHR using cannabis as compared with non-users. Furthermore, we explored the relationship between stress-induced PFC dopamine release and salivary cortisol response, attenuated psychotic symptoms, cognition, and pattern of cannabis use.

\section{2 \\ MATERIALS AND METHODS}

\subsection{Participants}

Thirty-six participants were initially enrolled and scanned $\left(72\left[{ }^{11} \mathrm{C}\right]\right.$ FLB457 PET scans), comprising 23 individuals at CHR-14 without concurrent cannabis use (referred as $\mathrm{CHR}$ ) and nine with concurrent cannabis use (referred as $\mathrm{CHR}-\mathrm{CU}$ )-and 13 matched $\mathrm{HV}$. One HV and one CHR-CU were excluded from the analysis because of excessive head motion that could not be corrected. The samples of HV and CHR without cannabis use included in this study were previously reported ${ }^{20}$ except one HV (age 38) who was removed from this analysis to better age-match the clinical groups.

To be eligible, all CHR individuals had to meet the following criteria: fulfillment of diagnostic criteria for prodromal syndrome as per the Criteria of Prodromal Syndromes (COPS) ${ }^{21}$ without substance use disorder (except cannabis in the CHR-CU group), as determined with the Structured Clinical Interview for DSM-5 $(\mathrm{SCID}-5)^{22}$ and no history of or current treatment with antipsychotic medication (antipsychotic-naïve). Additional inclusion criteria for $\mathrm{CHR}-\mathrm{CU}$ were regular cannabis use with a history of at least three times weekly for at least 2 months or meeting DSM-5 criteria for cannabis use disorder ${ }^{22}$ and positive drug screen for cannabis both at screening and on days of the PET scan. Furthermore, participants were asked not to use cannabis for 12 hours (overnight) prior to scanning to avoid being "high" during the stress task. HV did not meet criteria for any prodromal syndrome, had any history of psychiatric illness, recreational cannabis use (up to five times lifetime) or psychoactive drug use, and had no first-degree relative with a major mental disorder. Furthermore, HV and CHR were only eligible for enrolment with a negative urine drug screen for cannabis at the baseline visit.

Participants were excluded for any of the following: pregnancy or currently breastfeeding, clinically significant medical illness, and the presence of metal implants precluding a magnetic resonance imaging (MRI) scan.

The clinical status and severity of symptoms were assessed using the Structured Interview for Psychosis-risk Syndromes (SIPS) and the Scale of Psychosis-risk Symptoms (SOPS). ${ }^{21}$ Psychiatric status and brief drug history were assessed retrospectively with the SCID-5. A more detailed cannabis use pattern was assessed in $\mathrm{CHR}-\mathrm{CU}$ only using a semistructured interview after confirming cannabis use with a urine drug test. Cognitive function was assessed using the Repeatable Battery for the Assessment of Neuropsychological Status (RBANS) validated for investigating different cognitive domains in schizophrenia-related disorders such as immediate and delayed memory, visual-spatial abilities, verbal fluency, and attention.

This study was approved by the Research Ethics Board at the Centre for Addiction and Mental Health in accordance with the Declaration of Helsinki. All subjects provided written informed consent after being informed of all study procedures. 


\subsection{Montreal Imaging Stress Task}

Psychological stress was induced using the Montreal Imaging Stress Task (referred to as "stress"), ${ }^{23}$ which has been used and validated in various functional MRI and PET studies. ${ }^{20,23-25}$ In brief, subjects perform mental arithmetic presented on a computer screen that also displays information about the total number of errors, expected average number of errors, time spent on the current problem, and performance feedback for each problem (correct, incorrect, and time out). All subjects completed six blocks of arithmetic, each approximately 6 minutes in length, while lying in the scanner. The time constraint was adjusted individually to be slightly beyond each subject's abilities by adjusting each block dependent on the performance in the previous block. Because of this manipulation of the difficulty level, the average performance was set at $20 \%$ to $30 \%$ correct answers. Additionally, participants were given negative verbal feedback between each block, telling them that they need to improve their performance in order to reach minimum performance requirements. On a separate day before the stress session, participants were scanned while performing a Sensory Motor Control Task (referred to as "control"), using similar arithmetic but without any time constraints or negative verbal feedback. The control scan was always performed first, to avoid any residual effects of the stress task. In all experiments, the control or stress task was started about 6 to 8 minutes before tracer injection. The control task was also administered as a practice trial on a separate day before the PET experiments, to reduce novelty effects.

After each PET scan session, participants' subjective perception of stress was assessed by an abridged version (eight items) of the State Anxiety Questionnaire (SAQ). ${ }^{25}$ Further, subjects' attenuated psychotic symptoms were evaluated before and after each scan session using an abridged SOPS version.

\subsection{Physiological measures}

Saliva samples were collected every 15 minutes throughout the PET scanning session (six samples total) to evaluate the physiological response to the stress paradigm, starting 15 minutes before tracer injection and 9 minutes before the arithmetic task started. Saliva-derived cortisol was analysed using a time-resolved fluorescence immunoassay, and the normalized area under the curve $\left(A \cup C_{1}\right)$ (g/dL/min) was calculated for each subject and each PET scan session as described elsewhere. ${ }^{26}$ Normalization to time point 1 was chosen due to differences in the scan time (between 9.00 AM and 4.30 PM) as cortisol levels fluctuate over the course of the day. Change in $A \cup C_{1}\left(\triangle A \cup C_{1}\right)$ between control and stress task was defined as $\triangle A \cup C_{1}=$ $A U C_{1}$ Stress $-A U C_{I}$ Control. For further details, see (Schifani et $\mathrm{al}^{20}$ ).

\subsection{Image acquisition and reconstruction}

Image acquisition and reconstruction were described in detail before. ${ }^{20}$ In brief, every subject underwent a MRI scan to acquire a proton density-weighted image, used for delineation of individual regions of interest (ROIs) after coregistering with the PET image. All PET scans were performed for 90 minutes following intravenous bolus injection of approximately 305 to $410 \mathrm{MBq}\left[{ }^{11} \mathrm{C}\right] \mathrm{FLB} 457$ using a highresolution PET-CT scanner, Siemens-Biograph HiRez XVI (Siemens Molecular Imaging, Knoxville, TN, USA). Images were reconstructed using a 2D filtered back projection algorithm with a ramp filter at Nyquist cut-off frequency.

\subsection{PET data analyses}

Time-activity curves (TACs) were extracted for the dIPFC and mPFC including both hemispheres (Figure S1) and cerebellar cortex using our validated in-house imaging software ROMI. ${ }^{27}$ All ROIs were delineated using a proton density-weighted image for each participant. A quantitative estimate of binding was obtained from each TAC with the Simplified Reference Tissue Model (SRTM) using the in-house software $\mathrm{FMOD}$. The SRTM uses a within-brain reference region (cerebellar cortex in this case) instead of the arterial input function and provides an estimate of the binding potential $\left(B P_{N D}\right)$ of the radiotracer, which is proportional to the more fundamental parameters of receptor number $\left(B_{\max }\right)$ and affinity $\left(K_{d}\right)\left(B P_{N D} \propto B_{\max } / K_{d}\right)$. It is a validated method and commonly used with $\left[{ }^{11} \mathrm{C}\right]$ FLB457 (eg, Ito et $\mathrm{al}^{28}$, Narendran et $\mathrm{al}^{29}$, Olsson et $\left.\mathrm{al}^{30}\right)$. Although few studies suggest small specific binding of $\left[{ }^{11} \mathrm{C}\right]$ FLB457 in cerebellum, ${ }^{31,32}$ no change in cerebellar distribution volume was observed following challenges with amphetamine and methylphenidate. ${ }^{29,33}$ Previous studies with $\left[{ }^{11} \mathrm{C}\right]$ FLB457 have successfully used SRTM with cerebellum as a reference region (eg, Ko et $\mathrm{al}^{34}$, Mizrahi et $\mathrm{al}^{35}$ ), and a recent study showed that SRTM is a valid modelling approach to measure the percentage change in $B P_{N D}\left(\triangle B P_{N D}=1-B P_{N D}^{\text {Stress }} / B P_{N D}^{\text {Control }}\right)$ with $\left[{ }^{11} C\right] F L B 457 .{ }^{36}$ Right and left ROls were pooled together to create a single TAC used to derive the $B P_{N D}$. As quantifying $\left[{ }^{11} \mathrm{C}\right] \mathrm{FLB} 457$ is challenging, in part due to potential mass effects, a novel correction was applied in the current study, described in detail elsewhere. ${ }^{20}$ The corrected change in $B P_{N D}$ was calculated as first described by Gallezot et al ${ }^{37}$ :

$$
\Delta B P_{\mathrm{ND}}^{c}=\frac{\Delta B P_{\mathrm{ND}}\left(1+\left(\mu^{\text {Stress }} / E D_{50}\right)\right)+\left(\left(\mu^{\text {Control }}-\mu^{\text {Stress }}\right) / E D_{50}\right)}{1+\Delta B P_{\mathrm{ND}}\left(\mu^{\text {Stress }} / E D_{50}\right)+\left(\left(\mu^{\text {Control }}-\mu^{\text {Stress }}\right) / E D_{50}\right)}
$$

where $\mu$ is the ratio mass of radioligand injected to body weight and $E D_{50}$ the mass injected that would reduce $B P_{\mathrm{ND}}^{\text {Control }}$ by $50 \%$.

One CHR-CU participant moved his head significantly after 60 minutes of scan time during the stress scan, and these last 30-minute scan time were impossible to correct for motion. Therefore, we decided to only use the first 60 minutes of scan time data for analysis. As the Simplified Reference Tissue Model 2 (SRTM2) allows a more reliable calculation of $\left[{ }^{11} \mathrm{C}\right] \mathrm{FLB} 457 \mathrm{BP} \mathrm{ND}_{\mathrm{N}}$ for 60 -minute scan time data than the SRTM (see the Supporting Information for details on quantification with SRTM2 and Figures S2 and S3 for additional analyses), the quantitative estimate of binding was obtained from both (stress and control) TACs with SRTM2 instead of the SRTM for this participant. 


\subsection{Statistical analysis}

All statistical analyses were performed using SPSS version 22.0 (IBM, Armonk, NY, USA).

Group differences in stress-induced dopamine release were assessed using separate general linear models (GLMs) with $\triangle B P_{N D}$ value per ROI (dIPFC or $\mathrm{mPFC}$ ) as the dependent variable and group (CHR, CHR-CU, and HV) as the independent variable. To control for the effect of smoking, GLMs were rerun including smoking status as independent variable. Group differences in salivary cortisol response $\left(\triangle A \cup C_{1}\right)$ were assessed using a GLM with $\triangle A U C_{1}$ value as the dependent variable and group (CHR, CHR-CU, and HV) as the independent variable. All analyses were two-tailed with the conventional $\alpha=.05$. If significantly different, post hoc ANOVAs followed, using Bonferroni correction for multiple comparisons (three groups).

Changes in attenuated psychotic symptoms determined before and after the stress task were assessed using paired $t$ tests per group (CHR and $\mathrm{CHR}-\mathrm{CU}$ ). Group differences in attenuated psychotic symptoms following the stress task were assessed using Student's independent $t$ test.

Relationships between stress-induced dopamine release $\left(\triangle B P_{N D}\right)$ and salivary cortisol response $\left(\triangle A U C_{1}\right)$ or attenuated psychotic symptoms (abridged SOPS) were examined using separate GLMs with $\triangle B P_{N D}$ values per ROI (dIPFC or mPFC) as the dependent variable and group (CHR, $\mathrm{CHR}-\mathrm{CU}$, and $\mathrm{HV}$ ) as the independent variable while controlling for $\triangle A U C_{1}$ or for SOPS score, respectively, and including an interaction term between group and $\triangle A \cup C_{1}$ (for the associations with cortisol only). The main analyses were followed by Pearson's linear correlations.

Relationships between stress-induced dopamine release $\left(\triangle B P_{N D}\right)$ and cognition or cannabis use patterns were explored using GLMs or Pearson's linear correlations (see the Supporting Information for details). As these analyses were exploratory and carried out with limited power in this small sample, the results were not corrected for multiple comparisons and solely aimed to inform future studies.

We considered results to be significant at $P \leq .05$ and at trend levels at $P \leq .1$.

\section{$3 \quad$ RESULTS}

\subsection{Demographics and PET scan parameters}

Our final analysis comprised $14 \mathrm{CHR}$, eight CHR-CU, and $11 \mathrm{HV}$ (66 PET scans in total). Details of demographics, clinical and cannabis use characteristics, and scan parameters are summarized in Table 1. There were no significant differences between groups in sex, age, smoking status, and any of the PET scan parameters $(P>.05)$. The CHR-CU group had a significantly higher baseline SOPS positive and disorganized score than the CHR group (SOPS positive: $t=2.29$, .033; SOPS disorganized: $t=2.22, .039)$ and no difference in SOPS negative and general score $(P>.05)$.
Control and stress scans were performed on average $10.97 \pm$ 11.89 days apart. All subjects performed the tasks during the scans successfully.

\subsection{Scan paradigm effects}

As expected, SAQ revealed that all subjects felt less calm, satisfied, relaxed, and pleasant but more tense, strained, upset, and confused following the stress task than following the control task (Figure 1A; all $P<.0001$ ), suggesting that the stress paradigm was effective. Total SAQ scores (Figure 1B; positive items reversed scored) were significantly elevated in all groups following the stress as compared with the control task (effect of task: $F(1,30)=210.93, P<.0001$, Bonferroni-corrected $P<.0001$ for all groups). Furthermore, a significant group difference between SAQ scores was observed (effect of group: $F(2,30)=9.14, .0008$; for post hoc results, see caption of Figure 1) with no interaction between task and group $(F(2,30)=$ 1.42, .26).

All subjects performed significantly worse in the stress task (number of errors: $34.67 \pm 12.36[\mathrm{HV}], 36.30 \pm 9.81$ [CHR], and $36.15 \pm 7.82$ [CHR-CU]) than in the control task (number of errors: $4.55 \pm 2.22[\mathrm{HV}], 5.20 \pm 3.35[\mathrm{CHR}]$, and $5.44 \pm 2.62$ [CHR-CU]; effect of task: $F(1,30)=330.12, P<.0001$ ), showing that the stress task was able to adapt to the level of performance of each person and to produce a tailored programmed failure within each group.

\subsection{Stress-induced dopamine response in PFC}

$B P_{N D}$ at control conditions was not different among groups in any ROI (Figure S4; dIPFC: $F(2,30)=1.16, .33$; $\operatorname{mPFC}: F(2,30)=1.66$, .21). However, stress-induced dopamine release $\left(\triangle B P_{N D}\right)$ was significantly different among groups in mPFC (Figure 2; $F(2,30)=5.40$, .010) with $C H R-C U$ participants exhibiting lower $\triangle B P_{N D}$ compared with CHR participants (Bonferroni-corrected .008) but not compared with HV (Bonferroni-corrected .29). There was no difference among groups in the dIPFC (Figure 2; $F(2,30)=1.97, .16$ ). The same was true when removing the CHR-CU participant analysed with the SRTM2 for $\operatorname{mPFC}(F(2,29)=4.15, .026 ; \mathrm{CHR}$ vs CHR-CU: Bonferroni-corrected .026; HV vs CHR-CU: Bonferroni-corrected $.57)$ and $\operatorname{dIPFC}(F(2,29)=1.96, .16)$. Results remained similar when including smoking status as independent variable for mPFC (effect of group: $F(2,29)=5.20, .012$; $\mathrm{CHR}$ vs CHR-CU: Bonferronicorrected .010; HV vs CHR-CU: Bonferroni-corrected .35; effect of smoking status: $F(1,29)=0.001, .97$ ) and dIPFC (effect of group: $F(2,29)=1.69, .20$; effect of smoking status: $F(1,29)=0.20, .66)$. $\triangle B P_{N D}$ was also significantly lower in the CHR-CU group compared with $\mathrm{CHR}$ in $\mathrm{mPFC}$ when using the conventional calculation ${ }^{36}$ (without applying any correction for injected mass $\left[{ }^{11} \mathrm{C}\right] \mathrm{FLB} 457$ as per Gallezot ${ }^{37}$ (data not shown). 
TABLE 1 Participants' demographics, clinical characteristics, cannabis use characteristics, and radioligand injection parameters in a positron emission tomography (PET) study of dopamine release in healthy volunteers (HV), clinical high risk (CHR), and CHR with concurrent cannabis use (CHR-CU)

\begin{tabular}{|c|c|c|c|c|}
\hline & HV N = 11 & $\mathrm{CHR} N=14$ & CHR-CU N $=8^{b}$ & Comparisons \\
\hline \multicolumn{5}{|l|}{ Demographics } \\
\hline Gender (male/female) & $6 / 5$ & $6 / 8$ & $7 / 1$ & $\chi^{2}=4.91, .086$ \\
\hline Age, y (SD) & $24.91(5.54)$ & $22.07(3.38)$ & $22.13(2.80)$ & $F(2,30)=1.71, .20$ \\
\hline Smokers/nonsmokers & $1 / 10$ & $4 / 10$ & $3 / 5$ & $\chi^{2}=2.28, .32$ \\
\hline \multicolumn{5}{|l|}{ Clinical characteristics } \\
\hline SOPS positive symptoms (SD) & - & $10.71(3.45)$ & $13.88(2.36)^{\mathrm{a}}$ & $t=-2.29, d f=20, .033$ \\
\hline SOPS negative symptoms (SD) & - & $9.43(6.22)$ & $11.00(4.72)$ & $t=-0.62, d f=20, .54$ \\
\hline SOPS disorganized symptoms (SD) & - & $3.86(1.75)$ & $6.38(3.62)^{\mathrm{a}}$ & $t=-2.22, d f=20, .039$ \\
\hline SOPS general symptoms (SD) & - & $6.71(3.67)$ & $6.25(4.46)$ & $t=-0.26, d f=20, .79$ \\
\hline \multicolumn{5}{|l|}{ Cannabis characteristics } \\
\hline Age first cannabis use (SD) & N/A & $N / A$ & $15.88(2.15)$ & - \\
\hline Age first regular cannabis use (SD) & N/A & $\mathrm{N} / \mathrm{A}$ & $16.56(2.23)$ & - \\
\hline Months of cannabis use (SD) & N/A & $\mathrm{N} / \mathrm{A}$ & $66.44(53.65)$ & - \\
\hline Cumulative cannabis use occasions (SD) & $0.60(1.58)^{c}$ & $28.00(75.23)^{\mathrm{c}}$ & $4689.63(5507.17)$ & - \\
\hline \multicolumn{5}{|l|}{ PET parameters $\left(\left[{ }^{11} \mathrm{C}\right] \mathrm{FLB} 457\right)$} \\
\hline \multicolumn{5}{|l|}{ Amount injected, $\mathrm{MBq}(\mathrm{SD})$} \\
\hline Control task & $364.38(30.01)$ & $366.91(21.51)$ & $370.46(32.70)$ & $F(2,30)=0.11, .89$ \\
\hline Stress task & $380.53(20.23)$ & $372.06(21.32)$ & $362.37(25.52)$ & $F(2,30)=1.58, .22$ \\
\hline \multicolumn{5}{|l|}{ Specific activity, GBq/ $\mu \mathrm{mol}$ (SD) } \\
\hline Control task & $128.94(44.95)$ & $148.41(73.16)$ & $124.18(42.62)$ & $F(2,30)=0.56, .58$ \\
\hline Stress task & $148.32(63.60)$ & $120.79(60.78)$ & $125.41(33.95)$ & $F(2,30)=0.78, .47$ \\
\hline \multicolumn{5}{|l|}{ Mass injected, $\mu \mathrm{g}(\mathrm{SD})$} \\
\hline Control task & $1.14(0.30)$ & $1.11(0.47)$ & $1.22(0.41)$ & $F(2,30)=0.20, .82$ \\
\hline Stress task & $1.15(0.56)$ & $1.42(0.65)$ & $1.14(0.29)$ & $F(2,30)=1.03, .37$ \\
\hline
\end{tabular}

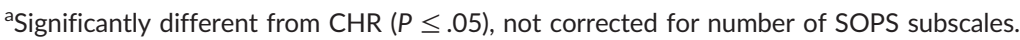

${ }^{\text {b}}$ Drug screen results for one participant were only available at baseline (but not at PET scan days), another participant was cannabis positive at baseline and control PET scan day but not at the stress scan day but was positive for alcohol, and a third participant was positive for benzodiazepines at the control PET scan day (in addition to cannabis).

'Three HV (one to five times lifetime) and five CHR (12-284 times lifetime) reported cannabis use history but history details were missing for one of the three HV.

Abbreviations: SD, standard deviation; SOPS, Scale of Psychosis-risk Symptoms.

(A)

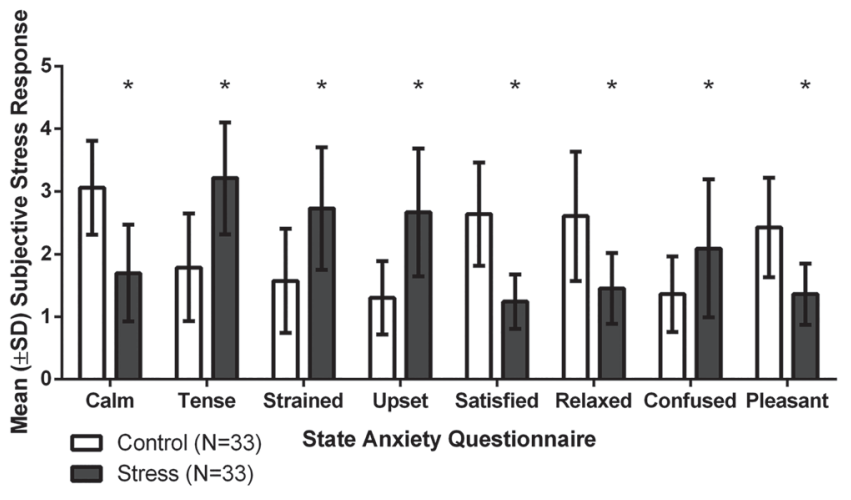

(B)

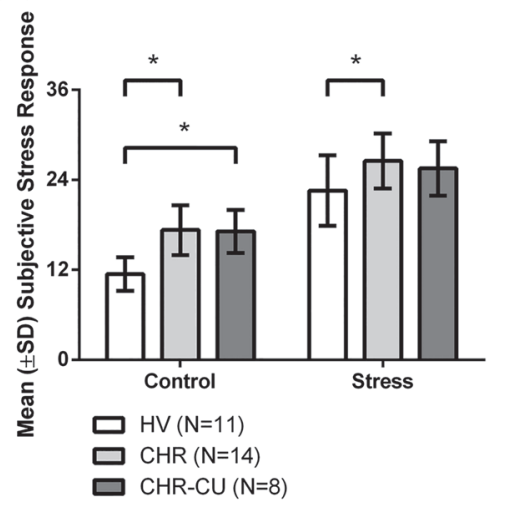

FIGURE 1 Subjective stress response following the control and stress task in healthy volunteers (HV), clinical high risk (CHR), and CHR with concurrent cannabis use (CHR-CU). Stress response was assessed with the state anxiety questionnaire in $\mathrm{A}$, individual categories for all subjects and $\mathrm{B}$, as total scores per group. ${ }^{*} P \leq .05$ (post hoc, after Bonferroni correction) 


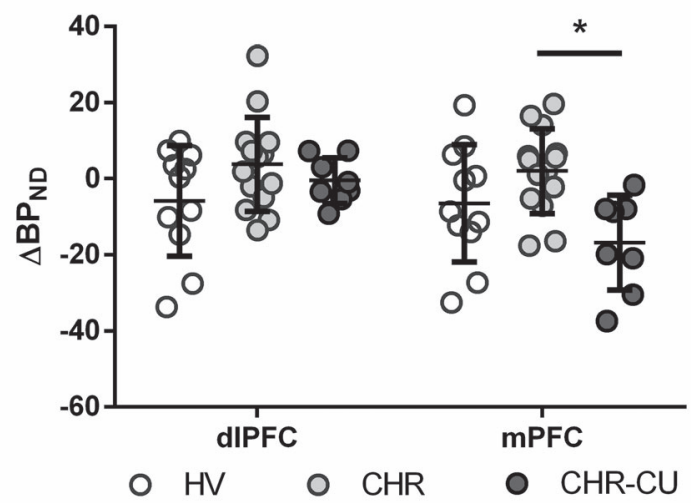

FIGURE 2 Difference in $\left[{ }^{11} \mathrm{C}\right] \mathrm{FLB} 457$ binding potential $\left(\triangle B P_{\mathrm{ND}}\right)$ in response to the stress task in healthy volunteers $(\mathrm{HV} ; \mathrm{N}=11)$, clinical high risk ( $\mathrm{CHR} ; \mathrm{N}=14)$, and $\mathrm{CHR}$ with concurrent cannabis use ( $\mathrm{CHR}-\mathrm{CU} ; \mathrm{N}=8$ ). Lines represent mean $\pm \mathrm{SD}$. ANOVA revealed a significant group effect in medial prefrontal cortex (mPFC; $F(2,30)=$ $5.40, .010$ ) but not dorsolateral prefrontal cortex (dIPFC; $P>.05$ ). ${ }^{*} P \leq$ .05 (post hoc, after Bonferroni correction)

\subsection{Salivary cortisol response to stress}

Salivary cortisol response $\left(\triangle A \cup C_{1}\right)$ was significantly different among groups (Figure 3A; $F(2,29)=5.08, .013$ ) with the CHR-CU participants exhibiting lower $\triangle A \cup C_{1}$ as compared with $C H R$ participants (Bonferronicorrected .018) but not compared with HV (Bonferroni-corrected 1.0). The CHR group had a slightly increased $\triangle A U C_{1}$ as compared with $H V$ (Bonferroni-corrected .087). Furthermore, we observed an overall positive relationship between $\triangle A U C_{1}$ and $\triangle B P_{N D}$ in $\mathrm{MPFC}$ (Figure 3B; omnibus test: $F(5,26)=6.39, .00054$; effect of $\triangle A \cup C_{1}$ on $\triangle B P_{N D}: F(1,26)=$ 6.29, .019) and dIPFC (Figure 3C; omnibus test: $F(5,26)=5.65, .0012$; effect of $\triangle A U C_{1}$ on $\left.\triangle B P_{N D}: F(1,26)=11.45, .0023\right)$, suggesting a direct relationship between dopamine release and salivary cortisol response due to the stress task. There were no interactions between group and $\triangle A \cup C_{1}$ neither in $\operatorname{mPFC}(F(2,26)=2.18 ; .13)$ nor $\operatorname{dIPFC}(F(2,26)=$ $1.10 ; .35$ ), suggesting no group differences in the relationship between dopamine release and salivary cortisol response due to the stress task.

(A)

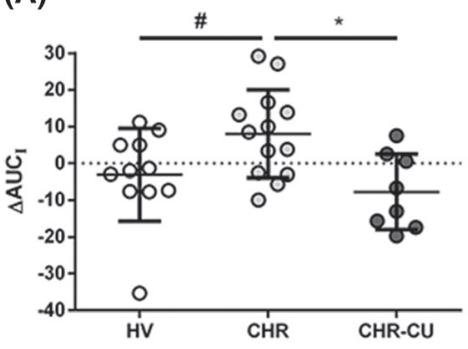

(B)

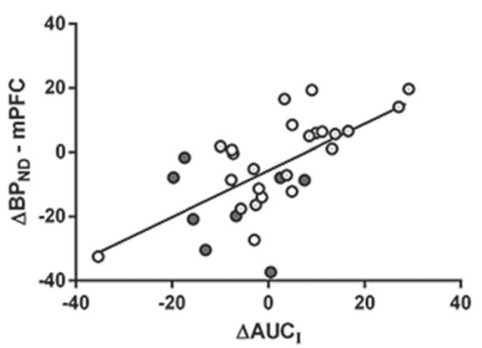

\subsection{Stress-induced change in attenuated psychotic symptoms}

CHR-CU individuals showed an increase in attenuated psychotic symptoms following the stress task (poststress) as compared with their score before the stress task (prestress) (Figure 4A; $t=4.58, d f=7$, .0025). No such difference was observed in CHR individuals $(t=$ $1.48, d f=12, .17)$. Additionally, CHR-CU individuals had higher attenuated psychotic symptoms following the stress task (poststress) as compared with CHR individuals ( $t=2.68, d f=19, .015$ ).

Furthermore, attenuated psychotic symptoms following the stress task (poststress) were negatively associated with $\triangle B P_{\mathrm{ND}}$ in $\mathrm{mPFC}$ (Figure 4B; omnibus test: $F(2,18)=9.35, .0016$; effect of abridged SOPS on $\triangle B P_{\mathrm{ND}}: F(1,18)=4.44, .049$ ) and dIPFC (Figure $4 \mathrm{C}$; omnibus test: $F(2,18)=4.65, .023$; effect of abridged SOPS on $\triangle B P_{\mathrm{ND}}$ : $F(1,18)$ $=8.43, .0095)$ across $\mathrm{CHR}$ and $\mathrm{CHR}-\mathrm{CU}$, suggesting that participants with more severe attenuated psychotic symptoms had lower PFC dopamine release in response to the stress task.

\subsection{Exploratory associations with cognitive assessments}

$\triangle B P_{\mathrm{ND}}$ in dIPFC was solely associated with scores of the immediate memory subscale of the RBANS, exclusively in CHR-CU individuals $(r=.83, .010)$ (see the Supporting Information for further information). This might suggest that CHR-CU individuals with lower immediate memory scores have lower stress-induced dopamine release in dIPFC, which needs to be replicated in future studies.

\subsection{Exploratory associations with cannabis use characteristics}

Given previous literature suggesting an inverse relationship with striatal dopamine release and frequency of cannabis use (days/ month), ${ }^{10}$ we explored the relationship between stress-induced cortical dopamine release and cannabis use characteristics. Characteristics

(C)

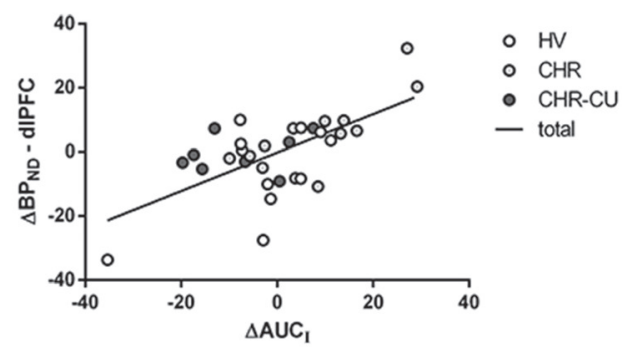

FIGURE 3 Salivary cortisol response $\left(\triangle A U C_{1}\right)$ in response to the stress task in healthy volunteers $(H V ; N=11)$, clinical high risk (CHR; $\left.N=13\right)$, and $\mathrm{CHR}$ with concurrent cannabis use (CHR-CU; $\mathrm{N}=8$ ). $\mathrm{A}$, The graph represents the difference in $\triangle A \cup C_{1}$ between control and stress task. Lines represent mean $\pm S D$. ANOVA revealed a significant group effect $(F(2,29)=5.08, .013) .{ }^{*} P \leq .05,{ }^{\#} P \leq .10$ (post hoc, after Bonferroni correction). ( $B$ and $C$ ) The line represents the best linear model fit of the associations between $\triangle A U C_{1}$ and $\left[{ }^{11} C\right] F L B 457 \triangle B P_{N D}$ in $B$, medial prefrontal cortex (mPFC; $r=.65, P<.0001$ ) or $C$, dorsolateral prefrontal cortex (dIPFC; $r=.64, P<.0001$ ) in the total sample including HV (open circles), CHR (light grey circles), and $\mathrm{CHR}-\mathrm{CU}$ (dark grey circles). Cortisol data from one $\mathrm{CHR}$ participant were not available for analysis. $A U C_{1}$, area under the curve; $B P_{\mathrm{ND}}$, binding potential 
(A)

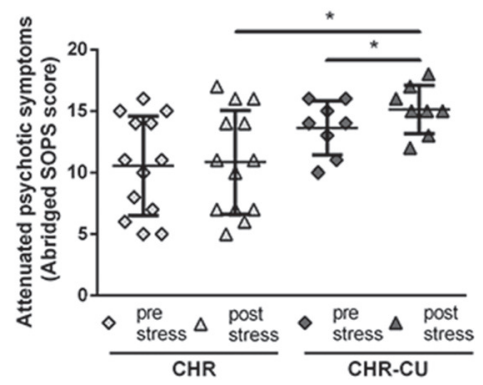

(B)

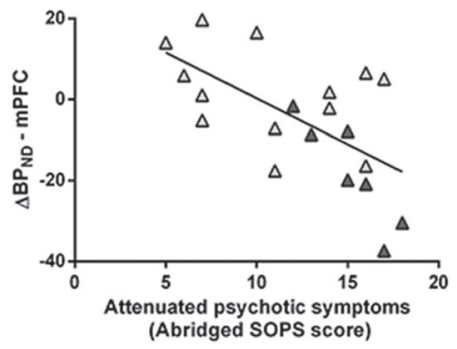

(C)

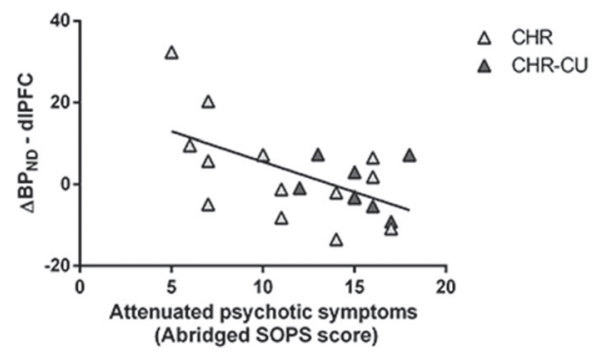

FIGURE 4 Attenuated psychotic symptoms in response to the stress task in clinical high risk (CHR; $\mathrm{N}=13$ ) and CHR with concurrent cannabis use (CHR-CU; $N=8$ ). A, The graph represents scores of the abridged Scale of Psychosis-risk Symptoms (SOPS) before and after the stress task. Lines represent mean $\pm S D$. ${ }^{*} P \leq .05$. (B and $C$ ) The line represents the best linear model fit of the associations between the score of the abridged SOPS after the stress task (poststress) and $\left[{ }^{11} \mathrm{C}\right] \mathrm{FLB} 457 \triangle B P_{\mathrm{ND}}$ in $\mathrm{B}$, medial prefrontal cortex (mPFC; $r=-.62 ; .0026$ ) or $\mathrm{C}$, dorsolateral prefrontal cortex (dIPFC; $r=-.57 ; .0073$ ) in the total high-risk sample including CHR (light grey triangles) and CHR-CU (dark grey triangles). Abridged SOPS score for one $\mathrm{CHR}$ participant was not available. $B P_{\mathrm{ND}}$, binding potential

of cannabis use history, such as length and age of first regular use, were inversely and positively associated with $\triangle B P_{\mathrm{ND}}$ in $\mathrm{mPFC}$, respectively, when controlling for current cannabis use. For statistics and details, see the Supporting Information.

Similar direction of results was obtained when we rerun all analyses including the HV (age 38), who was originally excluded from the group of $\mathrm{HV}$ in this paper to better age-match the CHR groups.

\section{DISCUSSION}

Here, we present, for the first time, results on cortical dopamine release in CHR who regularly use cannabis as compared with $\mathrm{CHR}$ non-users and HV. Our results show a decreased stress-induced dopamine release in the mPFC in CHR-CU participants as compared with $\mathrm{CHR}$. These results are in line with previous studies reporting a decreased stress-induced dopamine release in whole striatum and its subdivisions (including associative striatum, limbic striatum, and sensorimotor striatum) and in substantia nigra in CHR-CU individuals as compared with CHR. ${ }^{14,38}$ Similarly, individuals with a severe cannabis dependence presented blunted dopamine release in the same striatal regions upon amphetamine challenge as compared with non-users after a standardized abstinence of 5 days ${ }^{10}$ and healthy cannabis abusers showed blunted dopamine response in striatal regions upon methylphenidate challenge as compared with non-users ${ }^{11}$ (but see also Mizrahi et $\mathrm{al}^{12}$; Urban et $\mathrm{al}^{13}$ ).

There are a handful of studies investigating PFC dopamine function in regular cannabis users. For example, a study measuring glucose metabolism reported a lack in whole-brain glucose metabolism increase in female cannabis abusers upon methylphenidate challenge as compared with healthy female volunteers who showed an overall increase. $^{39}$ In drug users/abusers other than cannabis (including amphetamine, cocaine, methamphetamine, phencyclidine and alcohol), only one study measured PFC dopamine release in recently abstinent individuals with alcohol dependence using an amphetamine challenge. Similar to what we observed in the present study, Narendran et al ${ }^{40}$ showed significantly reduced dopamine release in mPFC (and other cortical regions) in alcohol-dependent subjects as compared with HV. We also observed a decreased stress-induced dopamine release in mPFC but not dIPFC. The difference in dopamine release between $\mathrm{PFC}$ regions might have been driven by the stress paradigm, as the $\mathrm{mPFC}$ is involved in stress regulation while the dIPFC is more involved in the regulation of cognitive function. ${ }^{15}$

Interestingly, in addition to the decreased mPFC dopamine release, the CHR-CU group exhibited reduced cortisol response as compared with non-using $\mathrm{CHR}$ participants, with an overall positive relationship between cortisol response and PFC (mPFC and dIPFC) dopamine release following stress. Such a disrupted stress response has been previously reported for healthy chronic cannabis users showing a blunted salivary cortisol stress reactivity to the Maastricht Acute Stress Test. ${ }^{41}$ The lower dopamine release in $\mathrm{mPFC}$ in regular cannabis users might partially explain deficits in emotional processing ${ }^{16}$ as the mPFC is strongly connected to subcortical regions that are key for emotional responses such as amygdala, hypothalamus, and hippocampus. ${ }^{15}$ In line with this, studies have reported a decrease in the blood oxygenation level dependent (BOLD) signal response in frontal cortex, cingulate, and amygdala during negative emotional stimuli presentation in heavy and regular cannabis users. ${ }^{42,43}$ In our earlier study, we did not observe any significant difference in stress-induced cortisol response between $\mathrm{CHR}$ and $\mathrm{CHR}-\mathrm{CU}$ participants, although $\mathrm{CHR}-\mathrm{CU}$ participants had a numerically lower mean cortisol response than CHR participants. Interestingly, striatal dopamine release and cortisol response following the stress task were only associated with one another in CHR but not in the CHR-CU group. ${ }^{14}$

Furthermore, the poststress attenuated psychotic symptoms were higher in CHR-CU as compared with CHR individuals and negatively associated with dopamine release in both PFC regions. This suggests that those subjects with lower dopamine release had higher stressinduced positive symptoms. This is in line with our recent findings that CHR individuals with higher distress and anxiety had lower PFC dopamine release ${ }^{20}$ and consistent with the suggestions that stress may precipitate psychosis in vulnerable individuals. ${ }^{44,45}$ 
The present results are especially important given the globally changing legal landscape for cannabis (reviewed by Hasin ${ }^{46}$ ). Chronic cannabis use has an especially deleterious effect when heavily used during adolescence, a key period for brain development and peak period of cannabis use. Especially in people with schizotypal personality traits and genetic vulnerability, an increased sensitivity to the acute psychotogenic effects of cannabis has been reported, and this has been shown to be a predictor of subsequent psychotic disorders (recently reviewed by Bloomfield et $\mathrm{al}^{47}$ ). Although discussions are ongoing regarding the risk of cannabis to induce psychosis in the general population, the available evidence strongly suggests an increased risk in susceptible individuals ${ }^{4}$ and in early heavy users. ${ }^{48}$ Therefore, more public and professional education about the risks of cannabis use and psychosis risk in susceptible individuals is of urgent necessity (discussed by Hasin ${ }^{46}$ ) and underscores the importance of the present findings.

\subsection{Limitations}

There are limitations inherent to neurochemical PET studies. First, the resolution of the PET scanner does not allow differentiation of histological subdivisions of MPFC and dIPFC (ie, ventromedial and dorsomedial PFC) and nearby structures. Second, the mass of $\left[{ }^{11} \mathrm{C}\right]$ FLB457 may not be at tracer dose. ${ }^{32}$ Therefore, we used a novel correction to account for this issue, ${ }^{20}$ first described by $\mathrm{Dr}$ Carlson and colleagues. ${ }^{37}$ When using the conventional calculation for $\triangle B P_{N D}$ (without applying this novel correction) with and without controlling for delta mass (stress-control conditions), results are similar. Third, the specific binding of $\left[{ }^{11} \mathrm{C}\right] \mathrm{FLB} 457$ in cerebellum may not be negligible, although its use as reference tissue in challenge-based experiments has been validated. ${ }^{36}$ We compared the cerebellar tracer uptake between both scans and showed nearly complete overlap (Figure S5). Fourth, since our control condition may be expected to recruit dopamine activity, ${ }^{49}$ it does not permit estimation of a true baseline $D_{2 / 3}$ receptor availability but serves as an excellent control for the cognitive aspect of the stress protocol. Fifth, our sample size of the CHR-CU group might seem small; however, an a priori sample size calculation using data on stress-induced striatal dopamine release in $\mathrm{CHR}$ and $\mathrm{CHR}-\mathrm{CU}$ published before ${ }^{14}$ demonstrated that seven participants per group would be needed to detect group effects (averaged effect size [Cohen's $d$ ] over all reported striatal region was 1.51; twotailed $t$ test at $\alpha=.05$ and $80 \%$ power). Similarly, a sample size calculation using the data on mPFC from the present study demonstrated that also seven participants per group would have been needed to detect group effects between CHR and CHR-CU groups (effect size [Cohen's d] for mPFC was 1.68; two-tailed $t$ test at $\alpha=.05$ and $80 \%$ power). Hence, it might be concluded that the used sample size of $14 \mathrm{CHR}$ and eight CHR-CU participants was enough to see an effect between those groups in mPFC. Sixth, although our sample size provides sufficient power to detect a group effect in stress-induced dopamine release $(n=33)$ and its associations with both salivary cortisol response $(n=33)$ and poststress positive symptoms $(n=22)$, the number of participants within each diagnostic group is small. Therefore, our exploratory associations between dopamine release and cognitive scores or cannabis use pattern are underpowered. These exploratory results (detailed in the Supporting Information) are only aimed to inform future studies. This, however, does not change our general conclusion. Seventh, a short abstinence (minimum of 12 hours in the current study) may have a potential direct effect of cannabis on our outcome measure. However, this is rather unlikely as studies measuring the effect of acute THC administration on dopamine transmission reported rather an increase (not a decrease) compared with nonusers. ${ }^{50}$ Eighth, it might be possible that a state of relative withdrawal may have contributed to the reduced dopaminergic response in the $\mathrm{CHR}-\mathrm{CU}$ group as participants were asked to stay abstinent from cannabis for 12 hours before scanning. Ninth, except for cumulative cannabis use occasions, no standardized information on cannabis use history/characteristics was available for HV and CHR participants. Although a history of cannabis use might impact dopamine release, ${ }^{10,11,14}$ the number of lifetime cannabis use occasions in HV and CHR in our study was extremely low as compared with CHR-CU participants (Table 1). Therefore, it is rather unlikely that the brief history of cannabis use in HV and CHR groups had any effect on the main outcome. Overall, while we acknowledge the limitations of both task and radioligand, these would not have been possible to overcome as (a) the stress task we used is the only validated one in PET imaging studies and (b) arterial sampling was impossible as all participants were doing the task with their hands while lying in the scanner. Thus, to date, there is no better methodology available to examine PFC dopamine response to a stress challenge in human.

\subsection{Conclusions and implication}

This study provides the first evidence of a blunted response to stress in PFC dopamine signalling in CHR-CU individuals, together with a reduced cortisol response. Cannabis use is increasingly perceived as relatively harmless and the prevalence of cannabis use disorder in youth is increasing (recently reviewed by Hasin ${ }^{46}$ ). Given the global trend to legalize cannabis (eg, in Canada as of October 2018) and the growing evidence of the increased risks for psychosis in vulnerable youth, this study is important as it highlights the effects of regular cannabis use on cortical dopamine function in youth at elevated risk for developing psychosis.

\section{ACKNOWLEDGMENTS}

We thank the staff of the Centre for Addiction and Mental Health (CAMH) Research Imaging Centre (Alvina Ng, Laura Nguyen, and Peter Bloomfield) and Focus on Youth Psychosis Prevention (FYPP) Clinic for their technical assistance, as well as Dr Efren Navas.

\section{AUTHOR CONTRIBUTIONS}

RM designed the study. CS, HHT, NR, AT, and JP conducted the experiments. CS, RM, and PR analysed the data. CS, PR, and RM wrote the manuscript. All authors critically reviewed the content and approved the final version for publication. 


\section{DISCLOSURE/CONFLICT OF INTEREST}

The authors declare no conflict of interest in relation to this work.

\section{FUNDING INFORMATION}

This work is partially supported by the operating grant "Stress-induced dopamine release in subjects at clinical high risk for psychosis: a $\left.{ }^{[11} \mathrm{C}\right]-$ FLB457 PET study" from the Canadian Institutes of Health Research (CIHR).

\section{ORCID}

Romina Mizrahi (1) https://orcid.org/0000-0001-6667-7928

\section{REFERENCES}

1. UNODC. World Drugs Report. Vienna: United Nations Publications; 2018.

2. Moore E, Mancuso SG, Slade T, Galletly C, Castle DJ. The impact of alcohol and illicit drugs on people with psychosis: the second Australian National Survey of Psychosis. Australian \& New Zealand J Psychiatry. 2012;46(9):864-878.

3. Rosen JL, Miller TJ, D'Andrea JT, McGlashan TH, Woods SW. Comorbid diagnoses in patients meeting criteria for the schizophrenia prodrome. Schizophr Res. 2006;85(1-3):124-131.

4. Murray RM, Quigley H, Quattrone D, Englund A, Di Forti M. Traditional marijuana, high-potency cannabis and synthetic cannabinoids: increasing risk for psychosis. World Psychiatry. 2016;15(3):195-204.

5. Marconi A, Di Forti M, Lewis CM, Murray RM, Vassos E. Meta-analysis of the association between the level of cannabis use and risk of psychosis. Schizophr Bull. 2016;42(5):1262-1269.

6. Large M, Sharma S, Compton MT, Slade T, Nielssen O. Cannabis use and earlier onset of psychosis: a systematic meta-analysis. Arch Gen Psychiatry. 2011;68(6):555-561.

7. Weinstein JJ, Chohan MO, Slifstein M, Kegeles LS, Moore H, AbiDargham A. pathway-specific dopamine abnormalities in schizophrenia. Biol Psychiatry. 2017;81(1):31-42.

8. Ashok AH, Mizuno Y, Volkow ND, Howes OD. Association of stimulant use with dopaminergic alterations in users of cocaine, amphetamine, or methamphetamine: a systematic review and meta-analysis. JAMA Psychiat. 2017;74(5):511-519.

9. Nutt DJ, Lingford-Hughes A, Erritzoe D, Stokes PR. The dopamine theory of addiction: 40 years of highs and lows. Nat Rev Neurosci. 2015;16(5):305-312.

10. van de Giessen E, Weinstein JJ, Cassidy CM, et al. Deficits in striatal dopamine release in cannabis dependence. Mol Psychiatry. 2017;22(1):68-75.

11. Volkow ND, Wang GJ, Telang F, et al. Decreased dopamine brain reactivity in marijuana abusers is associated with negative emotionality and addiction severity. Proc Natl Acad Sci U S A. 2014;111(30): E3149-E3156.

12. Mizrahi R, Suridjan I, Kenk M, et al. Dopamine response to psychosocial stress in chronic cannabis users: a PET study with $\left[{ }^{11} \mathrm{C}\right]-(+)-\mathrm{PHNO}$. Neuropsychopharmacol: Off Publ Amer Coll Neuropsychopharmacol. 2013;38(4):673-682.

13. Urban NB, Slifstein M, Thompson JL, et al. Dopamine release in chronic cannabis users: a $\left[{ }^{11} \mathrm{C}\right]$ raclopride positron emission tomography study. Biol Psychiatry. 2012;71(8):677-683.

14. Mizrahi R, Kenk M, Suridjan I, et al. Stress-induced dopamine response in subjects at clinical high risk for schizophrenia with and without concurrent cannabis use. Neuropsychopharmacol: Off Publ Amer Coll Neuropsychopharmacol. 2014;39(6):1479-1489.

15. Miller EK, Cohen JD. An integrative theory of prefrontal cortex function. Annu Rev Neurosci. 2001;24(1):167-202.

16. Hindocha C, Wollenberg O, Carter Leno V, Alvarez BO, Curran HV, Freeman TP. Emotional processing deficits in chronic cannabis use: a replication and extension. J Psychopharmacol. 2014;28(5):466-471.

17. Volkow ND, Swanson JM, Evins AE, et al. Effects of cannabis use on human behavior, including cognition, motivation, and psychosis: a review. JAMA Psychiat. 2016;73(3):292-297.

18. Holt DJ, Lebron-Milad K, Milad MR, et al. Extinction memory is impaired in schizophrenia. Biol Psychiatry. 2009;65(6):455-463.

19. Slifstein M, van de Giessen E, Van Snellenberg J, et al. Deficits in prefrontal cortical and extrastriatal dopamine release in schizophrenia: a positron emission tomographic functional magnetic resonance imaging study. JAMA Psychiat. 2015;72(4):316-324.

20. Schifani $C$, Tseng HH, Kenk $M$, et al. Cortical stress regulation is disrupted in schizophrenia but not in clinical high risk for psychosis. Brain. 2018;141(7):2213-2224.

21. Miller TJ, McGlashan TH, Rosen JL, et al. Prodromal assessment with the structured interview for prodromal syndromes and the scale of prodromal symptoms: predictive validity, interrater reliability, and training to reliability. Schizophr Bull. 2003;29(4):703-715.

22. First M, Williams J, Karg R, Spitzer R. Structured Clinical Interview for DSM-5: Research Version (SCID-5-RV). Arlington, VA: American Psychiatric Association; 2015.

23. Pruessner JC, Champagne F, Meaney MJ, Dagher A. Dopamine release in response to a psychological stress in humans and its relationship to early life maternal care: a positron emission tomography study using $\left[{ }^{11} \mathrm{C}\right]$ raclopride. J Neurosci. 2004;24(11):2825-2831.

24. Lederbogen F, Kirsch P, Haddad L, et al. City living and urban upbringing affect neural social stress processing in humans. Nature. 2011;474(7352):498-501.

25. Mizrahi R, Addington J, Rusjan PM, et al. Increased stress-induced dopamine release in psychosis. Biol Psychiatry. 2012;71(6):561-567.

26. Pruessner JC, Kirschbaum C, Meinlschmid G, Hellhammer DH. Two formulas for computation of the area under the curve represent measures of total hormone concentration versus time-dependent change. Psychoneuroendocrinology. 2003;28(7):916-931.

27. Rusjan P, Mamo D, Ginovart N, et al. An automated method for the extraction of regional data from PET images. Psychiatry Res. 2006;147(1):79-89.

28. Ito H, Sudo Y, Suhara T, Okubo Y, Halldin C, Farde L. Error analysis for quantification of $\left[{ }^{11} \mathrm{C}\right] \mathrm{FLB} 457$ binding to extrastriatal $\mathrm{D}(2)$ dopamine receptors in the human brain. Neurolmage. 2001;13(3):531-539.

29. Narendran R, Frankle WG, Mason NS, et al. Positron emission tomography imaging of amphetamine-induced dopamine release in the human cortex: a comparative evaluation of the high affinity dopamine D2/3 radiotracers $\left[{ }^{11} \mathrm{C}\right] \mathrm{FLB} \quad 457$ and $\left[{ }^{11} \mathrm{C}\right]$ fallypride. Synapse. 2009;63(6):447-461.

30. Olsson H, Halldin C, Swahn CG, Farde L. Quantification of [ $\left.{ }^{11} \mathrm{C}\right] \mathrm{FLB}$ 457 binding to extrastriatal dopamine receptors in the human brain. $J$ Cereb Blood Flow Metab. 1999;19(10):1164-1173.

31. Narendran R, Mason NS, Chen CM, et al. Evaluation of dopamine D2/3 specific binding in the cerebellum for the positron emission tomography radiotracer $\left[{ }^{11} \mathrm{C}\right] \mathrm{FLB}$ 457: implications for measuring cortical dopamine release. Synapse. 2011a;65(10):991-997.

32. Narendran R, Mason NS, May MA, et al. PET Imaging of dopamine D2/3 receptors in the human cortex with $\left[{ }^{11} \mathrm{C}\right] \mathrm{FLB}$ 457: reproducibility studies. Synapse. 2011b;65(1):35-40. 
33. Montgomery AJ, Asselin MC, Farde L, Grasby PM. Measurement of methylphenidate-induced change in extrastriatal dopamine concentration using $\left[{ }^{11} \mathrm{C}\right] \mathrm{FLB} 457$ PET. J Cereb Blood Flow Metab. 2007;27(2):369-377

34. Ko JH, Ptito A, Monchi O, et al. Increased dopamine release in the right anterior cingulate cortex during the performance of a sorting task: a $\left[{ }^{11}\right.$ C]FLB 457 PET study. Neurolmage. 2009;46(2):516-521.

35. Mizrahi R, Rusjan P, Agid O, et al. Adverse subjective experience with antipsychotics and its relationship to striatal and extrastriatal D2 receptors: a PET study in schizophrenia. Am J Psychiatry. 2007;164(4):630-637.

36. Sandiego CM, Gallezot JD, Lim K, et al. Reference region modeling approaches for amphetamine challenge studies with $\left[{ }^{11} \mathrm{C}\right] \mathrm{FLB} 457$ and PET. J Cereb Blood Flow Metab. 2015;35(4):623-629.

37. Gallezot JD, Planeta B, Nabulsi N, et al. Determination of receptor occupancy in the presence of mass dose: $\left[{ }^{11} \mathrm{C}\right]$ GSK189254 PET imaging of histamine H3 receptor occupancy by PF-03654746. J Cereb Blood Flow Metab. 2017;37(3):1095-1107.

38. Tseng $\mathrm{H}-\mathrm{H}$, Watts JJ, Kiang $\mathrm{M}$, et al. Nigral stress-induced dopamine release in clinical high risk and antipsychotic-naïve schizophrenia. Schizophr Bull. 2017;44:542-551.

39. Wiers CE, Shokri-Kojori E, Wong CT, et al. Cannabis abusers show hypofrontality and blunted brain responses to a stimulant challenge in females but not in males. Neuropsychopharmacol: Off Publ Amer Coll Neuropsychopharmacol. 2016;41(10):2596-2605.

40. Narendran R, Mason NS, Paris J, Himes ML, Douaihy AB, Frankle WG. Decreased prefrontal cortical dopamine transmission in alcoholism. Am J Psychiatry. 2014;171(8):881-888.

41. Cuttler C, Spradlin A, Nusbaum AT, Whitney P, Hinson JM, McLaughlin RJ. Blunted stress reactivity in chronic cannabis users. Psychopharmacology. 2017;234(15):2299-2309.

42. Gruber SA, Rogowska J, Yurgelun-Todd DA. Altered affective response in marijuana smokers: an FMRI study. Drug Alcohol Depend. 2009;105(1-2):139-153.

43. Zimmermann K, Walz C, Derckx RT, et al. Emotion regulation deficits in regular marijuana users. Hum Brain Mapp. 2017;38(8):4270-4279.
44. Corcoran C, Mujica-Parodi L, Yale S, Leitman D, Malaspina D. Could stress cause psychosis in individuals vulnerable to schizophrenia? CNS Spectr. 2002;7(1):33-42.

45. Corcoran C, Walker E, Huot R, et al. The stress cascade and schizophrenia: etiology and onset. Schizophr Bull. 2003;29(4):671-692.

46. Hasin DS. US epidemiology of cannabis use and associated problems. Neuropsychopharmacol: Off Publ Amer Coll Neuropsychopharmacol. 2018;43(1):195-212.

47. Bloomfield MA, Hindocha C, Green SF, et al. The neuropsychopharmacology of cannabis: a review of human imaging studies. Pharmacol Ther. 2018; 195: 132-161 https://doi.org/ 10.1016/j.pharmthera.2018.10.006

48. Hosseini S, Oremus M. The effect of age of initiation of cannabis use on psychosis, depression, and anxiety among youth under 25 years. Can J Psychiatr. 2018;706743718809339.

49. Egerton A, Mehta MA, Montgomery AJ, et al. The dopaminergic basis of human behaviors: a review of molecular imaging studies. Neurosci Biobehav Rev. 2009;33(7):1109-1132.

50. Bossong MG, Mehta MA, van Berckel BN, Howes OD, Kahn RS, Stokes PR. Further human evidence for striatal dopamine release induced by administration of 9-tetrahydrocannabinol (THC): selectivity to limbic striatum. Psychopharmacology. 2015;232(15):2723-2729. 